\title{
Diretrizes da Associação Brasileira de Estudos do Álcool e outras Drogas (ABEAD) para o diagnóstico e tratamento de comorbidades psiquiátricas e dependência de álcool e outras substâncias
}

\section{Guidelines of the Brazilian Association of Studies on Alcohol and Other Drugs (ABEAD) for diagnoses and treatment of psychiatric comorbidity with alcohol and other drugis dependence}

\begin{abstract}
Marcos Zaleski,, ${ }^{1,2}$ Ronaldo Ramos Laranjeira, ${ }^{3}$ Ana Cecilia Petta Roselli Marques, ${ }^{3}$ Lílian Ratto, ${ }^{4}$ Marcos Romano, ${ }^{3}$ Hamer Nastasy Palhares Alves, ${ }^{3}$ Márcia Britto de Macedo Soares, ${ }^{5}$ Valter Abelardino, ${ }^{6}$ Félix Kessler, ${ }^{7}$ Sílvia Brasiliano, ${ }^{8}$ Sérgio Nicastri, ${ }^{9}$ Patricia Brunferntrinker Hochgraf, ${ }^{8}$ Analice de Paula Gigliotti, ${ }^{10,11}$ Tadeu Lemos ${ }^{1,2}$

Resumo

O diagnóstico e tratamento de comorbidade psiquiátrica e dependência de álcool e outras substâncias tem sido objeto de inúmeros estudos nos últimos anos. A Associação Brasileira de Estudos do Álcool e Outras Drogas desenvolveu o projeto Diretrizes. Este trabalho visa o desenvolvimento de critérios diagnósticos e terapêuticos atualizados para as comorbidades psiquiátricas mais prevalentes. Ensaios clínicos randomizados, estudos epidemiológicos, com animais e outros estudos são revisados. As principais comorbidades psiquiátricas são estudadas e os dados de literatura resumidos, tendo como referência diretrizes adotadas em outros países. São abordados aspectos epidemiológicos, critérios diagnósticos, tratamento integrado e organização de serviço especializado, assim como especificidades do tratamento psicoterápico e farmacológico. As Diretrizes da Associação Brasileira de Estudos do Álcool e Outras Drogas reforçam a importância da abordagem adequada do dependente químico portador de comorbidade psiquiátrica.
\end{abstract}

Descritores: Transtornos relacionados ao uso de substâncias; Comorbidade; Diretrizes para a prática clínica; Diagnóstico; Terapia combinada

\section{Abstract}

Recently, several studies have focused on comorbity psychiatric disorders with alcohol and other substance dependence. The Brazilian Association of Studies on Alcohol and Other Drugs proposed the Brazilian Guidelines project. This study review diagnostic and therapeutic criteria to the most prevalent psychiatric comorbidities. Randomized clinical trials, epidemiological, animal studies and other forms of research are reviewed. The main psychiatric comorbidities are studied based on guidelines adopted by other countries and the literature data resumed. Epidemiological aspects, diagnoses, integrated treatment and service organization, as well as specific psychotherapic and pharmacological treatment are discussed. The Brazilian Association of Studies on Alcohol and Other Drugs Guidelines reassures the importance of adequate diagnoses and treatment regarding alcoholic and drug dependent patients suffering of comorbid psychiatric disorders.

Keywords: Substance related disorders; Comorbidity; Practice guidelines; Diagnosis; Combined modality therapy

1 Núcleo de Psiquiatria, Universidade Federal de Santa Catarina (UFSC), Florianópolis (SC), Brasil

2 Instituto de Psiquiatria de Santa Catarina, Florianópolis (SC), Brasil

3 Unidade de Pesquisa em Álcool e Drogas (UNIAD), Universidade Federal de São Paulo (UNIFESP), São Paulo (SP), Brasil

4 Unidade de Álcool e Drogas da Santa Casa de Misericórdia de São Paulo, São Paulo (SP), Brasil

5 Grupo de Estudos de Doenças Afetivas (GRUDA), Instituto de Psiquiatria, Hospital das Clinicas, Faculdade de Medicina, Universidade de São Paulo (USP), São Paulo (SP), Brasil

6 Centro de Atendimento Médico e Social (CAMPS) - Clínica Vitao, Curitiba (PR), Brasil

7 Equipe de Álcool e Drogas, Serviço de Psiquiatria, Hospital de Clínicas de Porto Alegre, Porto Alegre (RS), Brasil

8 Programa de Atenção à Mulher Dependente Química (PROMUD), Instituto de Psiquiatria, Hospital das Clínicas, Faculdade de Medicina, Universidade de São Paulo (USP), São Paulo (SP), Brasil

9 Programa Álcool e Drogas, Hospital Israelita Albert Einstein, São Paulo (SP), Brasil

10 Setor de Dependência Química, Santa Casa do Rio de Janeiro, Rio de Janeiro (RJ), Brasil

${ }^{11}$ Universidade Gama Filho, Rio de Janeiro (RJ), Brasil

Financiamento: Inexistente

Conflito de interesses: Inexistente

Recebido: 6 Outubro 2005

Aceito: 3 Novembro 2005
Correspondência

Marcos Zaleski

Rua Itapecerica,14 - Itacorubi

88034-420 Florianópolis, SC, Brasil

Tel: (55 48) 222-4218/ (55 48) 9972-6313

E-mail: mzaleski@terra.com.br 


\section{Introdução}

Comorbidade pode ser definida como a ocorrência de duas entidades diagnósticas em um mesmo indivíduo. No estudo da dependência de álcool e outras substâncias (AOS), a manifestação de transtornos mentais e de comportamento decorrentes do uso de drogas e de outros transtornos psiquiátricos vem sendo bastante estudada já desde os anos 80. ${ }^{1}$ De fato, o abuso de substâncias é o transtorno coexistente mais freqüente entre portadores de transtornos mentais, sendo de importância fundamental o correto diagnóstico das patologias envolvidas. Os transtornos mais comuns incluem os transtornos de humor, como a depressão, tanto uni como bipolar, transtornos de ansiedade, transtornos de conduta, déficit de atenção e hiperatividade e, numa extensão menor, a esquizofrenia. Transtornos alimentares e transtornos da personalidade também apresentam estreita correlação com o abuso de substâncias. ${ }^{2}$

Na última década, a co-ocorrência de transtornos mentais e transtornos devidos ao uso de substâncias psicoativas tem sido largamente reconhecida na clínica psiquiátrica. Diversos estudos, principalmente na Europa e nos Estados Unidos da América (EUA), relatam os efeitos negativos do uso/dependência de substâncias psicoativas entre pacientes com transtornos mentais, tentando estabelecer as potenciais diferenças entre pacientes que abusam de álcool ou substâncias psicoativas, principalmente nas implicações quanto a diagnóstico, tratamento e prognóstico. Há evidências de que mesmo o uso infreqüente e de pequenas doses de drogas, legais ou ilegais, podem levar o indivíduo com transtornos mentais graves a conseqüências mais sérias do que as vistas na população geral e está associados a mais efeitos negativos ligados aos transtornos mentais. ${ }^{3-4}$

\section{Epidemiologia}

Pacientes com comorbidade psiquiátrica em geral representam uma população heterogênea, tendo sido propostos muitos diferentes subtipos, baseados nas diferentes combinações entre os transtornos psiquiátricos existentes e as substâncias utilizadas, ou ainda conforme a idade de início do transtorno, gravidade do quadro e a duração de uso da substância e do transtorno mental. Muitos pacientes apresentam múltiplos transtornos psiquiátricos, uso de mais de uma substância e doenças clínicas associadas. ${ }^{5}$

A prevalência de comorbidade em dependência química varia conforme as diferenças no tipo de estudo realizado (se epidemiológico ou clínico), no tipo de serviço que deu origem às amostras, no método de avaliação utilizado, na definição do transtorno devido ao uso de substâncias, em variações nas características sociodemográficas das amostras, variações na disponibilidade de drogas ilícitas na comunidade e conforme a região geográfica estudada. ${ }^{6-8}$

Pesquisas do Epidemiologic Catchment Área Study (ECA) demonstraram que cerca de metade dos indivíduos diagnosticados com AOS pelos critérios do DSM-IV apresentam um diagnóstico psiquiátrico adicional: 26\% apresentam transtornos do humor, $28 \%$ transtorno de ansiedade, $18 \%$ transtornos da personalidade anti-social e $7 \%$ esquizofrenia. A prevalência de depressão maior entre dependentes químicos varia de 30 a $50 \% .^{9}$

Lotufo-Neto e Gentil avaliaram a prevalência de sintomas de ansiedade e fóbicos em pacientes dependentes de álcool, e também a prevalência de abuso e dependência de álcool entre aqueles que procuraram o serviço ambulatorial por diagnóstico de transtorno do pânico/agorafobia. ${ }^{10}$ Os resultados mostraram que os ataques de pânico e as fobias estavam associados com um aumento da gravidade e pior prognóstico para o alcoolismo, e que o abuso e dependência de álcool foi mais freqüente em homens com pânico e agorafobia que usavam o álcool para controlar os sintomas de ansiedade. Em outros estudos com pacientes com transtorno de ansiedade, 20 a 45\% relatam histórias de dependência de álcool. ${ }^{11-12}$

Existe a hipótese de que os indivíduos ansiosos acabam por usar o álcool como uma forma de automedicação, o que acaba por agravar o transtorno ansioso primário. ${ }^{13}$ Isto tem sido estudado e confirmado em alguns experimentos com animais. ${ }^{14}$

Portanto, transtornos ansiosos pré-mórbidos são considerados fatores de risco para o desenvolvimento de abuso e dependência de substâncias, assim como a ansiedade é um sintoma que faz parte da síndrome de abstinência e da intoxicação crônica por estas substâncias. ${ }^{15}$

A ocorrência de comorbidade com transtorno de déficit de atenção e hiperatividade (TDAH) e transtorno de conduta, que apresenta elevada incidência entre adolescentes com comorbidade com AOS, é maior entre adolescentes do sexo masculino do que do sexo feminino. ${ }^{16}$ Adultos com TDAH apresentam prevalência na vida muito maior para transtornos do uso de substâncias: aproximadamente 33\% dos adultos com TDAH apresentam antecedentes de abuso ou dependência de álcool e $20 \%$ deles história de abuso ou dependência de outras substâncias. ${ }^{17} \mathrm{O}$ uso de álcool é o mais prevalente entre adultos com TDAH, seguido pela maconha, estimulantes e cocaína. ${ }^{18}$

Uma revisão com estudos comunitários sobre AOS e comorbidade psiquiátrica revelou que $60 \%$ dos jovens com AOS tinham uma comorbidade, sendo o transtorno de conduta e o transtorno desafiador opositivo os mais comuns, seguidos pela depressão. ${ }^{19}$

Diversos trabalhos demonstram que a comorbidade com depressão com AOS é mais comum em mulheres do que em homens. ${ }^{20-22}$ Entre as mulheres com AOS, $19 \%$ tiveram depressão em algum momento na vida, enquanto que na população geral de mulheres essa prevalência é de 7\%. Nos homens com AOS, 78\% apresentaram primeiro AOS e depois depressão; entre as mulheres com AOS, 66\% apresentaram primeiro a depressão. ${ }^{9}$

Hendrick et al. também encontraram diferenças entre homens e mulheres no que se refere ao consumo de substâncias, em amostra clínica composta por 131 bipolares (63 mulheres e 68 homens). ${ }^{23}$ Neste estudo retrospectivo, os autores descreveram que homens bipolares apresentavam comorbidade com abuso/dependência de substâncias mais freqüentemente do que mulheres bipolares; essas, por sua vez, apresentavam problemas relacionados ao uso de álcool e de substâncias com maior freqüência do que mulheres da comunidade (freqüências 4 e 7 vezes maiores, respectivamente).

Estudos apontam para uma elevada prevalência de transtornos da personalidade e dependência de álcool e outras drogas. ${ }^{24}$ Abuso de uma ou mais substâncias foram relatados por $76 \%$ de pacientes com transtorno da personalidade borderline (TPB) e por $95 \%$ dos pacientes com transtorno da personalidade anti-social (TPAS), em adultos jovens internados em um serviço psiquiátrico de hospital geral. ${ }^{25}$

Para a comorbidade com esquizofrenia, o ECA demonstrou uma prevalência dos transtornos relacionados ao uso de AOS de aproximadamente $47 \%$, incluindo $34 \%$ com abuso de álcool e $28 \%$ com abuso de drogas. ${ }^{26-27}$ 
Em estudos de estimativas de prevalência com amostras clínicas de mulheres portadoras de algum tipo de transtorno alimentar, a associação com abuso ou dependência de álcool, presente ou passado, variou de 12 a 39\%, com uma média de $26 \%$. A associação entre bulimia e comportamentos bulímicos e quadros de dependência é mais forte do que em relação à anorexia. A porcentagem de bulímicas que relataram abuso ou dependência de álcool variou de 2,9 a 48,6\%, com uma média de 22,9\%.28 Em um estudo realizado na Universidade de São Paulo por Negrão e Cordás, ${ }^{29} 37,5 \%$ das pacientes que procuravam o ambulatório de transtornos alimentares faziam abuso de anfetaminas, e o mesmo percentual foi encontrado para aquelas que preenchiam critérios do DSM-IV para depressão maior. Já Bulik et al. compararam grupos de mulheres bulímicas sem história de dependência de álcool (BN-AD) com outro em que as mulheres preenchiam os critérios da DSM-III-R para dependência de álcool $(B N+A D) .{ }^{30} 0$ grupo $B N+A D$ apresentou aumento significativo do diagnóstico de comorbidade com transtorno da personalidade do grupo B, do número de tentativas de suicídio, uso diário de cigarros e consumo de outras substâncias psicoativas.

Pesquisas demonstram que a maioria dos pacientes alcoolistas comórbidos com tabagismo morrem de causas relacionadas ao tabagismo, o que revela a importância do tratamento desta comorbidade. ${ }^{31}$ Inúmeros estudos apareceram comprovando a associação de depressão maior com tabagismo, com taxas de prevalência entre 35 e $41 \%{ }^{32}$

A incidência de comorbidade com transtornos mentais graves parece estar aumentando. ${ }^{33}$ Tal fenômeno tem sido atribuído a uma maior disponibilidade de álcool e drogas na população geral. Alguns autores sugerem que é importante diferenciar pacientes com transtornos mentais graves que abusam de drogas psicoativas daqueles que apresentam quadros de dependência a essas drogas, uma vez que parece ser bastante diferente a evolução desses indivíduos. Parece haver uma tendência maior ao abandono do uso de álcool e drogas entre os pacientes que somente abusam delas do que naqueles que apresentam quadros de dependência. Isto mesmo apesar das evidências de que a prevalência de transtornos mentais por uso de substâncias psicoativas não seja muito variável entre uma população geral de pacientes durante a vida. ${ }^{34}$

\section{Diagnóstico}

Uma das maiores dificuldades na abordagem do paciente com comorbidade está no diagnóstico diferencial, pois ocorre uma superposição de sintomas. Um transtorno pode exacerbar ou mascarar o outro. Não é fácil, no início, estabelecer diferenças entre a presença de comorbidade psiquiátrica e abuso de substâncias psicoativas.

Por outro lado, também ainda não é claro o efeito dessas substâncias na apresentação dos sintomas em pacientes com transtornos mentais graves, não sendo possível estabelecer a real influência das drogas psicoativas sobre a psicopatologia: alucinações experimentadas por dependentes de álcool podem não diferir significativamente das alucinações experimentadas por pacientes esquizofrênicos. ${ }^{35}$ Aspectos envolvendo gênero, etnia e status socioeconômico também não devem ser esquecidos. Muitos autores concordam que tais fatores podem levar a pistas de situações ambientais traumáticas ou dificuldades variadas que influenciem o desenvolvimento e/ ou o agravamento, tanto das questões relacionadas ao abuso de substâncias quanto à comorbidade psiquiátrica. ${ }^{36} \mathrm{Em}$ razão do elevado índice de comorbidade com AOS entre mulhe- res que apresentam diagnóstico psiquiátrico, em relação aos homens, uma atenção especial deve ser dada para o acessamento de AOS para o sexo feminino. ${ }^{37}$

Muitos questionários têm sido aplicados à população de pacientes com transtornos mentais graves associados ao uso de drogas. A escolha da entrevista a ser utilizada deve basear-se nos objetivos do estudo, pois existem diferentes instrumentos desenvolvidos para cada tipo de avaliação especifica. Instrumentos de triagem servem para identificar indivíduos que provavelmente apresentem problemas relacionados ao abuso de substâncias; demandam maior sensibilidade que especificidade, como "CAGE" e o "AUDIT" (Alcohol Use Disorders Identification Test). ${ }^{38}$

O correto diagnóstico através das entrevistas iniciais ou da observação da evolução clínica pode facilitar a abordagem terapêutica e as estratégias de prevenção de recaída. Os estágios de mudança sugeridos por Prochaska e Di Clemente ${ }^{39}$ e amplamente empregados no tratamento da dependência química podem ser influenciados, por exemplo, por estados depressivos ou psicóticos.

\section{Tratamento}

1. Tratamento integrado e organização de serviço

Há cerca de uma década já existe um consenso entre os pesquisadores quanto ao sinergismo de sintomas. Muitos dos sintomas atribuídos a uma comorbidade são muitas vezes sintomas associados ao período de intoxicação ou de abstinência a uma ou mais substâncias.

Osher \& Kofoed propuseram abordagem integrada para pacientes comórbidos que incluem os seguintes fatores: estratégias para aumentar a aderência ao tratamento, persuasão acerca da relação entre abuso de substâncias e transtorno psiquiátrico e tratamento concomitante dos dois distúrbios para aliviar qualquer conflito entre as duas modalidades de tratamento. ${ }^{40}$ Outros autores também sugerem que o tratamento integrado de pacientes com comorbidade psiquiátrica tem um melhor resultado do que o tratamento seqüencial ou o paralelo, com uma abordagem abrangente, incluindo manejo da crise aguda por equipe multidisciplinar e por terapeuta individual, aguardando a desintoxicação com abstinência por no mínimo duas semanas. ${ }^{41-43} \mathrm{O}$ pior prognóstico dos pacientes dependentes químicos que apresentam comorbidade psiquiátrica pode ser atribuído, em grande parte, à abordagem tradicional, que trata a dependência em um serviço e o transtorno psiquiátrico associado em outro. ${ }^{41}$

Serviços voltados ao atendimento de pacientes dependentes têm pouca segurança e experiência em trabalhar com pacientes psicóticos, pacientes bipolares ou com graves transtornos de personalidade e acreditam que seu tratamento está além de suas possibilidades. ${ }^{44}$ Por esta razão, existem propostas para programas específicos, que permitam às equipes de saúde mental desenvolver formas efetivas de lidar com tais pacientes, visando conscientizá-los da necessidade de se tornarem abstinentes, melhorar sua aderência ao tratamento e reorganizar suas redes sociais. ${ }^{45}$ No Brasil, até o momento, são poucos os estudos que investigaram essa questão, mas há indicações de que a comorbidade entre transtornos mentais graves e abuso ou dependência de álcool ou drogas seja um problema relevante. ${ }^{46-48}$ Alguns autores enfatizam a necessidade de incluir no tratamento, além dos itens aqui citados, também programas psicoeducacionais para atendimento familiar. ${ }^{49}$ 


\section{Tratamento psicoterápico}

A entrevista motivacional (EM), um importante instrumento no tratamento de AOS, deve ser aplicada durante a fase inicial e durante o curso do tratamento de pacientes comórbidos. Pacientes com dependência química demandam maior esforço por parte do terapeuta para estabelecer uma aliança capaz de promover mudanças em seu comportamento e aumentar as possibilidades de aderência à terapia proposta. As psicoterapias têm se mostrado atualmente consistentes quando avaliadas em pesquisas clínicas para AOS e transtornos de ansiedade e do humor, tanto depressivo quanto bipolar, além de fortalecer a aliança terapêutica nos portadores de demais transtornos. ${ }^{50}$

Esta aliança tem importância especial para os portadores de transtorno da personalidade, que apresentam dificuldades para mudanças de estágio, redução da aderência e altas taxas de abandono de tratamento. Estudos mostram que estes pacientes se beneficiam do tratamento tanto quanto os que têm apenas diagnóstico em Eixo I, apesar de apresentarem recaídas mais precoces. ${ }^{51-52}$ Os transtornos de ansiedade e depressão também respondem bem às abordagens interpessoais, quando feitas por terapeutas experientes. ${ }^{53}$

Uma outra modalidade de psicoterapia, o tratamento psicossocial, deve ser aplicado imediatamente, pois pode determinar a utilização ou não da farmacoterapia em muitos casos e contribuir para diminuir as recaídas. Vários tipos de intervenção são preconizadas, dentre elas tem se dado preferência à terapia cognitivo-comportamental (TCC) e suas variantes, na forma de prevenção de recaída, tanto individual como em grupo. ${ }^{54-55}$

É consenso entre os pesquisadores que a comorbidade entre depressão e AOS intensifica os estados depressivos, diminui a resposta terapêutica e aumenta o risco de suicídio. ${ }^{56}$

Indivíduos com esquizofrenia e com abuso de substâncias têm um prognóstico pior do que pacientes com apenas um desses transtornos e são de difícil tratamento. Recentemente, vários guidelines têm sido propostos para o tratamento dos pacientes com essa comorbidade: o tratamento deve ser individualizado; o médico ou a equipe deve tentar diagnosticar a natureza da psicose, proteger o paciente dos danos próprios e alheios, bem como desintoxicá-lo e medicá-lo a fim de resolver os sintomas agudos. Ao contrário dos modelos de tratamento para dependência química, os grupos de auto-ajuda e o aconselhamento devem ter menor intensidade e poucas confrontações. ${ }^{57-58}$

São poucas as pesquisas sobre a eficácia terapêutica do tratamento integrado do transtorno alimentar com AOS. Em um dos poucos estudos em que foram incluídos pacientes dependentes com transtornos alimentares, el-Guebaly et al., com uma abordagem integrada em hospital-dia e focalizada nas necessidades individuais, observaram melhoras que foram sustentadas por um período superior a um ano, tanto no uso de álcool ou drogas quanto na qualidade de vida. ${ }^{59} 0$ tratamento de pacientes com comorbidade com transtornos alimentares tem como recursos, além do período de desintoxicação, uma combinação de técnicas que inclui prevenção de recaída para AOS, aconselhamento nutricional, intervenção psicossocial e tratamento farmacológico. ${ }^{59-60}$

Hughes demonstrou que o tratamento para parar de fumar em alcoolistas é tão bem sucedido quanto em não-alcoolistas. ${ }^{61}$ Em 1997, Stuyt comparou as taxas de recuperação entre fumantes e não-fumantes após tratamento para dependência de álcool e outras substâncias em regime de internação. ${ }^{62}$ Os resultados indicaram que não-fumantes apresentaram períodos mais longos de abstinência do que os fumantes. As diferenças encontradas são mais significativas em pacientes cuja droga de escolha atua como depressora do sistema nervoso central (ex: álcool). Não houve diferenças significativas das taxas de recuperação entre fumantes e nãofumantes quando se tratava de dependência de substâncias estimulantes (ex. cocaína).

A condução do tratamento do tabagismo pressupõe a modificação do padrão de uso de outras drogas, especialmente o álcool. Não há suporte científico para a noção tradicional de que a interrupção simultânea de álcool e nicotina possa aumentar o risco de recaída para o álcool. Na avaliação do paciente tabagista é fundamental observar o desejo de parar de fumar. Caso o paciente não deseje interromper o uso do tabaco, deve-se tratar a comorbidade e utilizar estratégias psicoterápicas para motivá-lo (entrevistas motivacionais). ${ }^{63}$

\section{Tratamento farmacológico}

A regra geral é aguardar o período de desintoxicação para iniciar o tratamento da comorbidade. Obviamente, se um paciente está ativamente psicótico, agressivo ou suicida, intervenção imediata específica deve ser empreendida, ainda que se considere o transtorno afetivo relacionado à dependência química (ou seja, farmacoterapia, proteção ambiental, orientação familiar, psicoterapia suportiva).

O tratamento deve seguir as orientações para cada entidade psiquiátrica, associando, quando necessário, o uso dos agentes anticraving naltrexona para reduzir da impulsividade, e do acamprosato para reduzir a reação ao estresse e sensibilidade a sintomas de ansiedade, agentes estes aprovados apenas para dependência de álcool. ${ }^{64-65}$

No entanto, diversos estudos sugerem cuidados especiais na orientação farmacológica de algumas das comorbidades com os transtornos psiquiátricos aqui abordados, e que serão discutidos a seguir.

Em todos os casos de comorbidades psiquiátricas com AOS há restrições ao uso de benzodiazepínicos (BZD), devido ao risco de tolerância e dependência cruzada com AOS. ${ }^{66-67} \mathrm{~A}$ buspirona é a substância ansiolítica mais recomendada, considerada tão segura e efetiva como o BZD. ${ }^{68}$

Para o TDAH, autores divergem quanto ao uso seguro do metilfenilato, em razão do potencial de abuso por sua ação dopaminérgica. ${ }^{69-70}$ Estudos recentes demonstram que a bupropiona apresenta-se como uma opção mais segura e igualmente eficaz, embora mais estudos sejam necessários para definição desta substância como sendo de primeira escolha no tratamento da comorbidade com AOS. ${ }^{71-72}$

Para a comorbidade com depressão (e também para os comórbidos com transtorno da personalidade e transtorno alimentar que apresentam sintomas depressivos), estudos demonstram que os tricíclicos causam melhora nos sintomas e diminuem as taxas de recaída, mas seus efeitos colaterais produzem altos índices de abandono. ${ }^{73}$ Diversos estudos demonstram a eficácia de antidepressivos tricíclicos e dos inibidores de recaptação de serotonina para o tratamento destes pacientes, levando à redução da freqüência e severidade das recaídas. ${ }^{56,74}$ Embora inibidores da MAO possam ser utilizados, devem ser cuidadosamente considerados os riscos de recaída a AOS e comportamento de ingestão alimentar descontrolado (risco de ingestão de alimentos ricos em tiramina). ${ }^{60}$

A pequena margem de segurança para o uso do lítio torna difícil o seu uso com segurança para os pacientes que 
apresentam THB e abuso de AOS (baixa aderência, seguimento descontínuo, dificuldade em manter estado de hidratação continuamente satisfatório - o que aumenta o risco de intoxicação por hemoconcentração). ${ }^{75}$ Assim, há uma maior preferência pelo uso de anticonvulsivantes como o valproato e a carbamazepina. $^{76}$

Algumas vezes os neurolépticos podem exacerbar os sintomas psicóticos ou causar delirium, devendo ser priorizados nos estágios mais avançados do tratamento, quando os efeitos das substâncias psicoativas tiverem desaparecido. A farmacoterapia com neurolépticos é indicada na menor dose possível. Estudos recentes demonstram uma preferência pelo uso de neurolépticos atípicos para comórbidos com transtornos psicóticos. Isto ocorre tanto por sua ação mais eficaz sobre sintomas negativos, o que reduz o abuso de drogas como tentativa de pseudo-tratar os sintomas deficitários dos quadros psicóticos crônicos, quanto pela ação anticraving de algumas destas substâncias, como a olanzapina, clozapina e quetiapina. ${ }^{77-78}$

\section{Considerações gerais finais}

Os seguintes itens a serem considerados, centrados em estratégias de manejo biopsicossocial: ${ }^{79}$

1) Considerar a combinação específica da comorbidade e o estágio de motivação ao escolher o melhor método de tratamento.

2) Considerar o uso de farmacoterapia para o tratamento do transtorno psiquiátrico, desintoxicação e fase inicial de recuperação e prevenção de recaída.

3) Usar técnicas psicossociais para aumentar a motivação, auxiliar na resolução de problemas ambientais e no manejo de situações difíceis.

4) Fornecer apoio familiar e informação sobre tratamento adicional de apoio, como grupos baseados nos 12 passos de Alcoólicos Anônimos e outros grupos de auto-ajuda.

5) Apoio psiquiátrico para o controle de sintomas psicóticos, mania e depressivos com ou sem risco de suicídio.

Em resumo, a Figura 1 apresenta os critérios para uma abordagem adequada ao paciente com diagnóstico de transtorno psiquiátrico e dependência de álcool e outras drogas.

\section{HISTÓRIA FAMILIAR}
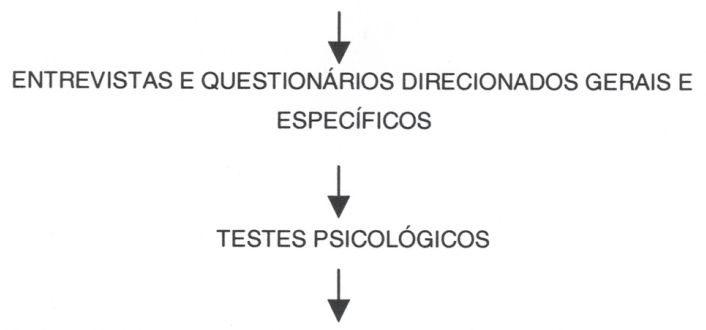

DIAGNÓSTICO DIFERENCIAL COM USO DA CID-10 OU DSM-IV

TRATAMENTO INTEGRADO PARA TRANSTORNO INDUZIDO
POR SUBSTÂNCIA E COMORBIDADE PSIQUIÁTRICA
ABORDAGEM BIOPSICOSSOCIAL PARA TRATAMENTOE
PREVENÇÃO DE RECAÍDA

Figura 1 - Comorbidade psiquiátrica e dependência de álcool e outras substâncias. Critérios de acessamento

\section{Referências}

1. Ross HE, Glaser FB, Germanson T. The prevalence of psychiatric disorders in patients with alcohol and other drug problems. Arch Gen Psychiatry. 1988;45(11):1023-31.

2. Watkins TR, Lewellen A, Barret M. Issues and problems with dual diagnosis. In: Dual diagnosis: An integrated approach to treatment. Thousand Oaks, Ca: Sage; 2001. p. 11-24.

3. Drake RE, Osher FC, Wallach MA. Alcohol use and abuse in schizophrenia. A prospective community study. J Nerv Ment Dis. 1989;177(7):408-14.

4. Swofford CD, Kasckow JW, Scheller-Gilkey G, Inderbitzin LB. Substance use: a powerful predictor of relapse in schizophrenia. Schizoph Res. 1996;20(1-2):145-51.

5. Ziedonis D, Brady K. Dual diagnosis in primary care. Detecting and treating both the addiction and the mental illness. Med Clin North Am. 1997;81(4):1017-36.

6. Regier DA, Boyd JH, Burk JD Jr, Rae DS, Myers JK, Kramer M, George LK, Robins LN, Karno M, Locke BZ. One-month prevalence of mental disorders in the United States. Based on five Epidemiologic Catchment Area sites. Arch Gen Psychiatry. 1988;45(11):977-86.

7. Drake RE, Wallach MA. Moderate drinking among people with severe mental illness. Hosp Community Psychiatry. 1993;44(8):780-1.

8. el-Guebaly N. Substance abuse and mental disorders: the dual diagnostic concept. Can J Psychiatry. 1990;35(3):261-7.

9. Hersh DF, Modesto-Lowe V. Drug abuse and mood disorders. In: Henry BK, Bruce J, Rounsaville MD, editors. Dual Diagnosis and treatment: Substance Abuse and Comorbid Medical and Psychiatric Disorders. New York: Marcel Dekker; 1998. p. 177-201.

10. Lotufo-Neto F, Gentil, V. Alcoholism and phobic anxiety-a clinicaldemographic comparison. Addiction. 1994;89(4):447-53.

11. Cox BJ, Norton GR, Swinson RP, Endler NS. Substance abuse and panic-related anxiety: a critical review. Behav Res Ther. 1990;28(5):385-93.

12. Kushner MG, Sher KJ, Beitman BD. The relation between alcohol problems and the anxiety disorders. Am J Psychiatry. $1990 ; 147(6): 685-95$.

13. Kranzler HR, Mason B Modesto-Lowe V. Prevalence, diagnosis, and treatment of comorbid mood disorders and alcoholism. In: Kranzler HR, Rounsaville B, eds. Dual Diagnosis and Treatment. New York: Marcel Dekker, Inc; 1998. p. 107-36.

14. Hitzemann R. Animal models of psychiatric disorders and their relevance to alcoholism. Alcohol Res Health. 2000;24(3):149-58.

15. Schuckit MA, Monteiro MG. Alcoholism, anxiety and depression. $\mathrm{Br}$ J Addiction. 1988;83(12):1373-80.

16. Latimer WW, Stone AL, Voight A, Winters KC, August GJ. Gender differences in psychiatric comorbidity among adolescents with substance use disorders. Exp Clin Psychopharmacol. 2002;10(3):310-5.

17. Biederman J, Faraone SV, Spencer T, Wilens T, Norman D, Lapey KA, Mick E, Lehman BK, Doyle A. Patterns of psychiatric comorbidity, cognition, and psychosocial functioning in adults with attention deficit hyperactivity disorder. Am J Psychiatry. 1993;150(12): 1792-8.

18. Biederman J, Wilens T, Mick E, Milberger S, Spencer TJ, Faraone SV. Psychoactive substance use disorders in adults with attention deficit hyperactivity disorder (ADHD): effects of ADHD and psychiatric comorbidity. Am J Psychiatry. 1995;152(11):1652-8.

19. Armstrong TD, Costello EJ. Community studies on adolescent substance use, abuse, or dependence and psychiatric comorbidity. J Consult Clin Psychol. 2002;70(6):1224-39.

20. King AC, Bernardy NC, Hauner K. Stressful events, personality, and mood disturbance: gender differences in alcoholics and problem drinkers. Addict Behav. 2003;28(1):171-87.

21. Brienza RS, Stein MD. Alcohol use disorders in primary care: do gender-specific differences exist? J Gen Intern Med. 2002; 17(5):387-97.

22. Johnson ME, Yep MJ, Brems C, Theno SA, Fisher DG. Relationship among gender, depression, and needle sharing in a sample of injection drug users. Psychol Addict Behav. $2002 ; 16(4): 338-41$. 
23. Hendrick V, Altshuler LL, Gitlin MJ, Delrahim S, Hammen C. Gender and bipolar illness. J Clin Psychiatry. 2000;61(5):393-6; quiz 397

24. Rounsaville BJ, Kranzler HR, Ball S, Tenne H, Poling J, Trifflemann E. Personality disorders in substance abusers: relation to substance use. J Nerv Ment Dis. 1998;186(2):87-95.

25. Hatzitaskos P, Soldatos CR, Kokkevi A, Stefanis CN. Substance abuse patterns and their association with psychopathology and type of hostility in male patients with borderline and antisocial personality disorder. Compr Psychiatry. 1999;40(4):278-82.

26. Regier DA, Myers JK, Kramer M, Robins LN, Blazer DG, Hough RL, Eaton WW, Locke BZ. The NIMH Epidemiological Catchment Area Program. Historical context, major objectives, and study population characteristics. Arch Gen Psychiatry. 1984;41(10):934-41.

27. Regier DA, Farmer ME, Rae DG, Locke BZ, Keith SJ, Judd LL, Goodwin FK. Comorbidity of mental disorders with alcohol and other drug abuse. Results from the Epidemiological Catchment Area (ECA). Study. JAMA. 1990;264(19):2511-18.

28. Holderness CC, Brooks-Gunn J, Warren MP. Co-morbidity of eating disorders and substance abuse. Int J Eating Disorder. 1994;16(1):1-34. Review.

29. Negrao AB, Cordas TA. Clinical characteristics and course of anorexia nervosa in Latin America, a Brazilian sample. Psychiatry Res. 1996;62(1):17-21

30. Bulik CM, Sullivan PF, Carter FA, Joyce PR. Lifetime comorbidity of alcohol dependence in women with bulimia nervosa. Addict Behav. 1997;22(4):437-46.

31. Hurt RD, Offord KP, Croghan IT, Gomez-Dahl L, Kottke TE, Morse RM, Melton LJ 3rd. Mortality following inpatient addictions treatment. Role of tobacco use in a community-based cohort. JAMA. 1996;275(14): 1097-103. Erratum: JAMA. 1996;276(10):784.

32. Glassman AH, Helzer JE, Covey LS, Cottler LB, Steiner F, Tipp JE, Johnson J. Smoking cessation and major depression. JAMA. 1990;264(12): 1546-49.

33. Cuffel BJ. Prevalence estimates of substance abuse in schizophrenia and their correlates. J Nerv Ment Dis. 1992;180(9):589-92, discussion 593-6. Review.

34. Bartels SJ, Drake RE, Wallach MA. Long-term course of substance use disorders among patients with severe mental illness. Psychiatr Serv. 1995;46(3):248-51

35. Salloum IM, Moss HB, Daley DC. Substance abuse and schizophrenia: impediments to optimal care. Am J Drug Alcohol Abuse. 1991;17(3):321-36.

36. Patrick DL, Bergner M. Measurement of health status in the 1990's. Ann Rev Public Health. 1990;11:165-83.

37. Chander G, McCaul ME. Co-occurring psychiatric disorders in women with addictions. Obstet Gynecol Clin North Am. 2003;30(3):469-81

38. Babor TF, de la Fuente JR, Saunders J, Grant M. AUDIT: the alcohol use disorders identification test: guidelines for use in primary health care. Geneva: World Health Organization; 1992.

39. Prochaska JO, Di Clemente CC. Toward a comprehensive model of change. In: Miller WR, Heather N, eds. Treating addictive behaviors: processes of change. New York: Plenum; 1986. p. 3-27.

40. Osher FC, Kofoed LL. Treatment of patients with psychiatric and psychoactive substance abuse disorders. Hosp Community Psychiatry. 1989;40(10):1025-30.

41. Ryglewicz H, Pepper B. The dual disorder client: mental disorder and substance use. In: Cooper S, Lentner TH, eds. Innovations in community mental health. Sarasota, FL: Professional Research Press; 1992. p. 270-90.

42. Ries RK, Ellingson T. A pilot assessment at one month of 17 dual diagnoses patients. Hosp Community Psychiatry. 1990;41(11):1230-3.

43. Drake RE, Wallach MA. Moderate drinking among people with severe mental illness. Hosp Community Psychiatry. 1993;44(8): 780-2.

44. Johnson S. Dual diagnosis of severe mental ilness and substance misuse: a case for specialist services? $\mathrm{Br} J$ Psychiatry. 1990;171:205-8.
45. Jerrel JM, Ridgely MS. Comparative effectiveness of three approaches to serving people whit severe mental illness and substance abuse disorders. J Nerv Ment Dis. 1995;183(9):566-76.

46. Menezes PR. The outcome of schizophrenia and related psychoses after hospitalization: a prospective study in São Paulo, Brazil [thesis]. London: University of London; 1995.

47. Ratto LRC. Prevalência da comorbilidade entre transtornos mentais graves e transtornos devidos ao uso de substâncias psicoativas em São Paulo, Brasil [dissertação]. São Paulo: Faculdade de Medicina da Universidade de São Paulo; 2000. p. 74.

48. Heckert U, Andrade L, Alves MJ, Martins C. Lifetime prevalence of mental disorders among homeless people in a southeast city in Brazil. Eur Arch Psychiatry Clin Neurosci. 1999;249(3):150-5.

49. Links PS. Developing effective services for patients with personality disorders. Can J Psychiatry. 1998;43(3):251-9.

50. Beck AT, Wright FD, Newman CF, Liese BS. Cognitive therapy of substance abuse. New York: Guilford Press; 1993. p. 340.

51. Reich JH, Vasile RG. Effect of personality disorders on the treatment outcome of Axis I conditions: an update. J Nerv Ment Dis. 1993;181(8):475-84. Review.

52. Strand, JG, Benjamin LS. Resistance to change in individuals with personality disorders. Curr Opin Psychiatry. 1997;10(2):132-5.

53. Fleck MP, Lafer B, Sougey EB, Del Porto JA, Brasil MA, Juruena MF. Diretrizes da Associação Médica Brasileira para o tratamento da depressão (versão integral). Rev Bras Psiquiatr. 2003;25(2): 114-22.

54. Schuckit MA. Drug and alcohol abuse. 4th ed. New York: Plenum Publishing; 1995.

55. Marlatt GA, Gordon JR. Relapse prevention. New York: Guilford; 1985.

56. Cornelius JR, Bukstein O, Salloum I, Clark D. Alcohol and psychiatric comorbidity. Recent Dev Alcohol. 2003;16:361-74.

57. Bradizza CM, Stasiewicz PR. Integrating substance abuse treatment for the seriously mentally ill into inpatient psychiatric treatment. $J$ Subst Abuse Treat. 1997;14(2):103-11.

58. Ries RK, Russo J, Wingerson D, Snowden M, Comtois KA, Srebnik D, Roy-Byrne P. Shorter hospital stays and more rapid improvement among patients with schizophrenia and substance disorders. Psychiatr Serv. 2000;51(2):210-5.

59. el-Guebaly N, Hodgins DC, Armstrong S, Addington J. Methodological and clinical challenges in evaluating treatment outcome of substance-related disorders and comorbidity. Can J Psychiatry. 1999;44(3):264-70.

60. Yager J, Andersen A, Devlin M, Egger H, Herzog D, Mitchell J, Powers P, Yates A, Zerbe K, eds. Practice guidelines for the treatment of patients with eating disorders. 2nd ed. Washington DC: American Psychiatric Association Press; 2000.

61. Hughes JR. Treatment of smoking cessation in smokers with past alcohol/drug problems. J Subst Abuse Treat. 1993;10(2):182-7.

62. Stuyt EB. Recovery rates after treatment for alcohol/drug dependence. Tobacco users vs. non-tobacco users. Am J Addict. 1997;6(2):159-67.

63. Bien TH, Burge R. Smoking and drinking: a review of the literature. Int J Addict. 1990;25(12):1429-54.

64. Farren CK, O'Malley SS. Occurrence and management of depression in the context of naltrexone treatment of alcoholism. Am J Psychiatry. 1999;156(8):1258-62.

65. Tedeschi M. Naltrexone and acamprosate. Using new drugs for alcohol dependence. Aust Fam Physician. 2001;30(5):447-50.

66. Busto U, Sellers EM, Naranjo CA, Cappell HD, Sanchez-Craig M, Simpkins J. Patterns of benzodiazepine abuse and dependence. $\mathrm{Br}$ J Addict. 1986;81(1):87-94.

67. Ellis P, Carney MW. Benzodiazepines abuse and management of anxiety in the community. Int J Addict. 1988;23(10):1083-90.

68. Kranzler HR, Burleson JA, Del Boca FK, Babor TF, Korner P, Brown $\mathrm{J}$, Bohn MJ. Buspirone reatment of anxious alcoholics. A placebocontrolled trial. Arch Gen Psychiatry. 1994;51(9):720-31.

69. Schubiner H, Tzelepis A, Isaacson JH, Warbasse LH 3rd, Zacharek M, Musial J. The dual diagnosis of attention-deficit/hyperactivity disorder and substance abuse: case reports and literature review. $J$ Clin Psychiatry. 1995;56(4):146-50.

70. Volkow ND, Fowler JS, Wang GJ, Ding YS, Gatley SJ. Role of dopamine in the therapeutic and reinforcing effects of methylphenidate in 
humans: results from imaging studies. Eur Neuropsychopharmacol. 2002;12(6):557-66.

71. Levin FR, Evans SM, McDowell DM, Brooks DJ, Nunes E. Bupropion treatment for cocaine abuse and adult attention-deficit/hyperactivity disorder. J Addict Dis. 2002;21(2):1-16.

72. Riggs PD, Leon SL, Mikulich SK, Pottle LC. An open trial of bupropion for ADHD in adolescents with substance use disorders and conduct disorder. J Am Acad Child Adolesc Psychiatry. 1998;37(12):1271-8.

73. Anderson IM, Nutt DJ, Deakin JF. Evidence-based guidelines for treating depressive disorders with antidepressants: a revision of the 1993 British Association for Psychopharmacology guidelines. British Association for Psychopharmacology. J Psychopharmacol. 2000;14(1):3-20.

74. Rossinfosse C, Wauthy J, Bertrand J. SSRI antidepressants and alcoholism.Rev Med Liege. 2000;55(11):1003-10.

75. Nunes EV, McGrath PJ, Wager S, Quitkin FM. Lithium treatment for cocaine abuser with bipolar spectrum disorders. Am J Psychiatry. 1990;147(5):655-7.

76. Sonne SC, Brady KT. Substance abuse and bipolar comorbidity. Psychiat Clin North Am. 1999;22:609-27. ix. Review.

77. Hutchison KE, Wooden A, Swift RM, Smolen A, McGeary J, Adler L, Paris L. Olanzapine reduces craving for alcohol: a DRD4 VNTR polymorphism by pharmacotherapy interaction. Neuropsychopharmacology. 2003;28(10):1882-8.

78. Potvin S, Stip E, Roy JY. Clozapine, quetiapine and olanzapine among addicted schizophrenic patients: towards testable hypotheses. Int Clin Psychopharmacol. 2003;18(3):121-32.

79. Ziedonis D, Brady K. Dual diagnosis in primary care. Detecting and treating both the addiction and the mental illness. Med Clin America. 1997;81(4):1017-36. 